\title{
Cardiac Arrest Following Foreign-Body Aspiration
}

\author{
Sally Cheukying Wong MB/BChir and Syed Mohammad Tariq FRCP
}

\section{Introduction}

Foreign-body aspiration is common in children but less frequent in adults, and in adults the presentation is often more delayed than in children. ${ }^{1}$ Aspiration of food, despite causing immediate cough, may not be reported by an adult, as the cough usually subsides quickly once the foreign body lodges in a more distal airway. This is attributed to the larger caliber of adult airways, so the obstruction is often more distal and therefore less likely to cause asphyxiation. ${ }^{1}$

\section{Case Summary}

A previously well and fully independent female, age 73 years, was admitted with a right basal pneumonia and new-onset atrial fibrillation. She had no history of cardiac disease. She had good response to treatment with antibiotics and digoxin, but on the fourth hospital day she suffered a cardiac arrest while having her lunch. The nursing staff immediately put out an "arrest call" and started cardiac massage and bag-and-mask ventilation. The resident on call for intensive care promptly intubated the patient. The initial rhythm on cardiac monitor showed pulseless electrical activity. Following intravenous epinephrine (1 $\mathrm{mg}$ in $10 \mathrm{~mL}$ ), cardiac rhythm changed to ventricular fibrillation. After one electric shock of $360 \mathrm{~J}$, she reverted to sinus rhythm with good cardiac output. The total duration of cardiopulmonary resuscitation was recorded as $4 \mathrm{~min}$. Therapeutic hypothermia was not given, because at that time it was not available in our intensive care unit.

Sally Cheukying Wong MB/BChir is affiliated with the Respiratory Unit, Queen Elizabeth Hospital, King's Lynn, Norfolk, United Kingdom. Syed Mohammad Tariq FRCP is affiliated with the Respiratory Unit, Luton and Dunstable Hospital, Luton, United Kingdom.

The authors have disclosed no conflicts of interest.

Correspondence: Syed M Tariq FRCP, Respiratory Unit, Luton and Dunstable Hospital, Lewsey Road, Luton LU4 0DZ United Kingdom. E-mail: doc_smt@hotmail.com.

DOI: $10.4187 /$ respcare.00766
A cardiac cause for the arrest was suspected, but the post-arrest electrocardiogram was normal. She had no history of cardiac disease and no recognized risk factors for pulmonary embolism. A portable chest radiograph taken after resuscitation (Fig. 1) showed the right basal consolidation and suggested a left-lower-lobe collapse, with straightening of the left heart border, a loss of definition of the left hemidiaphragm, and blunting of the left costophrenic angle.

The clues to the foreign-body aspiration were the timing of the arrest (while she was having lunch), the presence of new chest radiographic features consistent with left-lowerlobe collapse, and a high peak inflation pressure (up to $45 \mathrm{~cm} \mathrm{H}_{2} \mathrm{O}$ ) required for mechanical ventilation after resuscitation. After transferring her to the intensive care unit, a fiberoptic bronchoscopy, through the endotracheal tube, revealed an inhaled cube of boiled sweet potato (Fig. 2) that was blocking the left-lower-lobe bronchus, which was removed with a wire basket. The wire basket holding the foreign body, the bronchoscope, and the endotracheal tube were removed en bloc, followed by re-intubation. The inflation pressure stabilized to $<30 \mathrm{~cm} \mathrm{H}_{2} \mathrm{O}$ after removing the foreign body and restoring the patency of the leftlower-lobe bronchus. A chest radiograph after the bronchoscopy showed re-expansion of the left lower lobe.

Serum troponin I level 12 hours after the cardiac arrest was $0.032 \mathrm{ng} / \mathrm{mL}$ (normal range $<0.04 \mathrm{ng} / \mathrm{mL}$ ). She remained in sinus rhythm after resuscitation, hence the digoxin was discontinued. The next day her swallowing was assessed after extubation and found to be normal. She had an uneventful recovery from the cardiac arrest, with no apparent neurocognitive impairment. She was discharged home after a week in the hospital.

\section{Discussion}

In this patient there were several clues to foreign-body aspiration. The first clue was the high post-resuscitation peak inflation pressure (up to $45 \mathrm{~cm} \mathrm{H}_{2} \mathrm{O}$ ). Second, chest radiograph showed a new left basal collapse from occlusion of the left-lower-lobe bronchus. Third, the cardiac arrest occurred while she was having her lunch. A cardiac cause for the arrest was considered, mainly because of the 


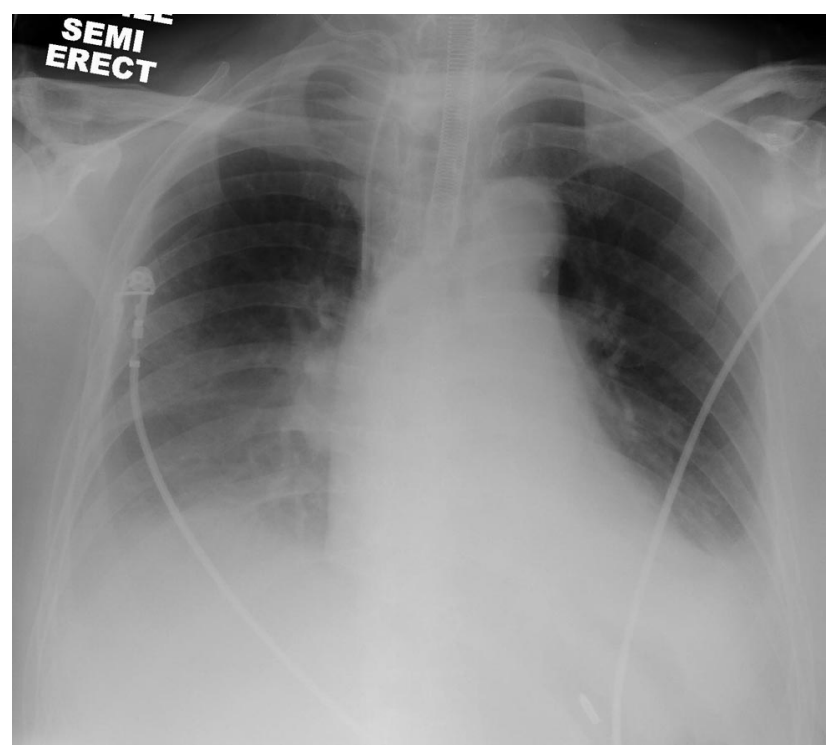

Fig. 1. Antero-posterior portable chest radiograph shows right basal consolidation and features of left-lower-lobe collapse, evidenced by straightening of the left heart border and blunting of the left costophrenic angle. Note the endotracheal tube and right central venous catheter in position.

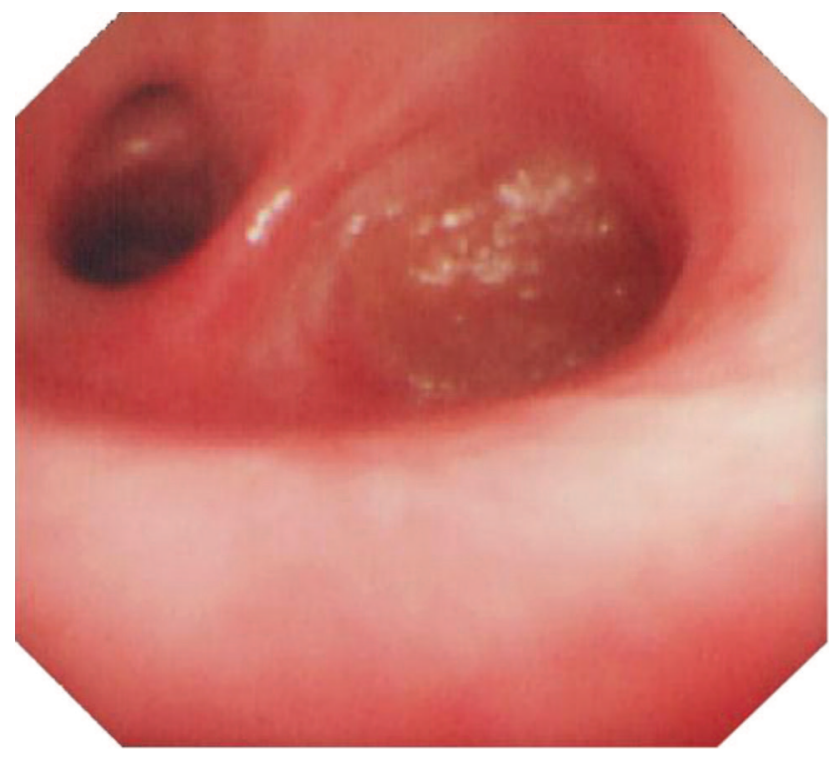

Fig. 2. Bronchoscopy shows the aspirated foreign body (a cube of boiled sweet potato) blocking the left-lower-lobe bronchus.

new-onset atrial fibrillation complicating her right basal pneumonia, but she had no history of coronary artery disease. In addition, her electrocardiogram and the serum troponin I, 12 hours after arrest, excluded an acute cardiac event. We therefore believe the arrest resulted from asphyxia due to the aspirated foreign body initially blocking her trachea. The foreign body was subsequently pushed into the left-lower-lobe bronchus when she was intubated and ventilated.
Table 1. Risk Factors for Foreign-Body Aspiration

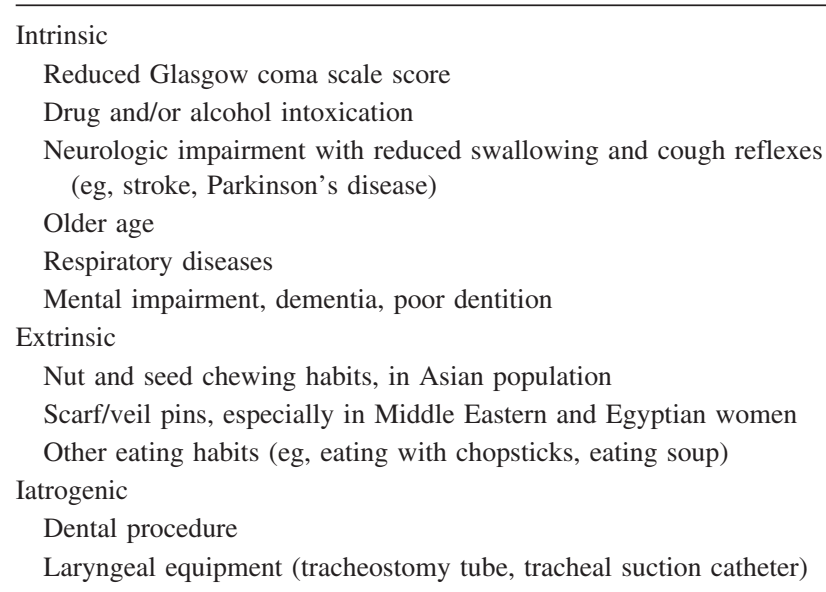

The incidence of sudden death from food asphyxiation is relatively low. An older study of hospitalized adult patients, however, found food asphyxiation as a cause of death in 14 of $1,087(1.3 \%)$ autopsies performed over 5 years. $^{2}$ Those patients died suddenly, during or shortly after meals. Old age (12 of 14 were $>64$ y old), poor dentition, and sedation were notable risk factors. We believe that in our patient, apart from old age, her cough caused by the community-acquired pneumonia was an important factor contributing to foreign-body aspiration.

Asphyxia from foreign-body aspiration in adults is uncommon, because items small enough to pass through the vocal cords are often too small to obstruct the main airway and cause asphyxia. ${ }^{3,4}$ Published reports of death or near death in adults due to foreign-body aspiration have generally been associated with altered consciousness, old age, or a rapidly expanding foreign body, such as a sucralfate tablet. ${ }^{2,4,5}$ The impactions in those cases were mainly at the main bronchus level. ${ }^{4,5}$

A broad range of aspirated foreign bodies has been reported, including food and organic items such as peanuts, vegetable seeds, and meat bone, and inorganic objects such as veil/scarf pins and pen caps. ${ }^{1,5}$ Food items are usually the aspirated foreign bodies in adults, especially the elderly. ${ }^{1,5,6}$ The literature on foreign-body aspiration in adults suggests 3 categories of risk factor (Table 1)., ${ }^{1,2,4-9}$ Chen et al postulated that in patients with respiratory comorbidities the increased risk of aspirating food items may be related to the raised respiratory rate, causing difficulty with swallowing. A blunting of protective reflexes secondary to hypercapnia may also play a role. ${ }^{5} \mathrm{~A}$ further likely risk, as in our patient, could be an uncontrollable bout of cough while eating.

Chest radiograph is usually non-specific unless the foreign body is radiopaque (eg, metal or bone). Atelectasis, lobar pneumonia, bronchiectasis, and fibrosis are associated with foreign-body aspiration, especially when the di- 
agnosis is delayed. However, it may be normal to detect no new radiographic abnormality. ${ }^{1,7}$ Computed tomography has been used in some reported cases, often as a diagnostic tool when the history is atypical or to ascertain the location of the foreign body and the severity of secondary lung damage, such as bronchiectasis or bronchostenosis. ${ }^{7}$

Aspirated foreign bodies are more often found in the right side than the left, and in the lower rather than the upper airways, ${ }^{5,8}$ probably because the right main bronchus is shorter, larger in diameter, and at a less acute angle to the trachea than the left main bronchus. Interestingly, some studies have found no significant difference in right versus left side for aspirated foreign bodies in pediatric patients..$^{1,7}$

Several studies have advocated flexible bronchoscopy as the first-line treatment for foreign-body aspiration in adults. ${ }^{1,5,9}$ Chen et al reported a success rate of $58 \%$ on first attempt and $74 \%$ overall. ${ }^{5}$ This was thought to be operator-dependent. ${ }^{1}$ However, rigid bronchoscopy is helpful for larger objects that cannot be retrieved with a flexible bronchoscope, so a rigid bronchoscope should be available as a backup. ${ }^{9}$ If neither type of bronchoscopy allows retrieval, surgery may be needed, especially where severe irreversible secondary lung damage is evident, requiring a lobectomy or pneumonectomy. ${ }^{1,5}$

Therapeutic hypothermia, whether by surface cooling or by cold intravenous infusion, is a relatively new intervention after successful cardiopulmonary resuscitation. ${ }^{10,11}$ Hypothermia provides organ protection, especially to the brain, and reduces the risk of cognitive impairment. We did not have hypothermia facilities at the time of the abovedescribed patient. Reassuringly, our patient was resuscitated promptly, her condition stabilized rapidly after removal of the foreign body, and she had no discernible neurocognitive impairment at discharge. We believe she had an un-witnessed food aspiration that caused asphyxia and cardiac arrest. Her episodic cough and persistent tachypnea related to her community-acquired pneumonia had increased her risk of foreign-body aspiration. The resuscitation and intubation probably dislodged the food item from the trachea and pushed it into the left-lower-lobe bronchus. The airway obstruction caused by the foreign body accounted for the post-intubation high inflation pressure and the new radiograph changes consistent with leftlower-lobe collapse.

Prompt diagnosis of foreign-body aspiration is crucial, as serious complications such as pneumonia and abscess formation in the lung distal to the foreign body and subsequent progression to bronchiectasis can be avoided if the foreign body is retrieved early. Identification of other risk factors (eg, impaired swallowing) is important to prevent further aspirations. Foreign-body aspiration should always be considered as a possible diagnosis in patients with car- diorespiratory arrest, especially in patients who were eating immediately prior to the arrest, and who require unexpectedly high inflation pressure to ventilate after resuscitation. Relatively high inflation pressure can also be due to reduced compliance in poorly ventilated lungs after arrest. Urgent bronchoscopy can be invaluable in identifying and treating such cases.

\section{Teaching Points}

- Cardiac arrest or life-threatening asphyxiation after foreign-body aspiration is more common in children than adults.

- Food items are common aspirated foreign bodies in adults, especially the elderly.

- Risk factors for foreign-body aspiration include respiratory diseases, especially those that cause dyspnea or episodic cough, and conditions that impair consciousness or swallowing.

- Clues to foreign-body aspiration include the timing of the event (coincidental with eating), new radiographic features of lung or lobar collapse, and high peak inflation pressure during mechanical ventilation.

- Prompt bronchoscopic retrieval of the foreign body minimizes the risk of long-term sequelae.

\section{REFERENCES}

1. Baharloo F, Veyckemans F, Francis C, Biettlot MP, Rodenstein DO. Tracheobronchial foreign bodies: presentation and management in children and adults. Chest 1999;115(5):1357-1362.

2. Irwin RS, Ashba JK, Braman SS, Lee HY, Carrao WM. Food asphyxiation in hospitalized patients. JAMA 1977;237(25):2744-2745.

3. Overdahl MC, Wewers MD. Acute occlusion of a mainstem bronchus by a rapidly expanding foreign body. Chest 1994;105(5):16001602.

4. Pritt B, Harmon M, Schwartz M, Cooper K. A tale of 3 aspirations: foreign bodies in the airway. J Clin Pathol 2003;56(10):791-794.

5. Chen CH, Lai CL, Tsai TT, Lee YC, Perng RP. Foreign body aspiration into the lower airway in Chinese adults. Chest 1997;112(1): 129-133.

6. Zubairi AB, Haque AS, Hussain SJ, Khan JA. Foreign body aspiration in adults. Singapore Med J 2006;47(5):415-418.

7. Sersar SI, Hamza UA, Abdel Hameed WA, Abdul Maaty RA, Gowaeli NN, Moussa SA, et al. Inhaled foreign bodies: management according to early and late presentation. Eur J Cardiothorac Surg 2005; 28(3):369-374.

8. Limper AH, Prakash UB. Tracheobronchial foreign bodies in adults. Ann Intern Med 1990;112(8):604-609.

9. Tariq SM, George J, Srinivasan S. Inhaled foreign bodies in adolescents and adults. Monaldi Arch Chest Dis 2005;63(4):193-198.

10. Nolan JP, Soar J. Postresuscitation care: entering a new era. Curr Opin Crit Care 2010;16(3):216-222.

11. Arrich J, Holzer M, Herkner H, Mullner M. Hypothermia for neuroprotection in adults after cardiopulmonary resuscitation. Cochrane Database Syst Rev 2009;(4):CD004128. 